Rev. Inst. Flor. v. 27 n. 2 p. $137-143$ dez. 2015

http://dx.doi.org/10.4322/rif.2015.010

ISSN impresso 0103-2674/on-line 2178-5031

\title{
SELEÇÃO DE CLONES DE Hevea brasiliensis PARA A REGIÃO DE MOCOCA, ESTADO DE SÃO PAULO ${ }^{1}$ CLONE SELECTION OF Hevea brasiliensis TO MOCOCA REGION, STATE OF SÃO PAULO
}

\author{
Israel Luiz de LIMA ${ }^{2,9}$; Hugo Rodrigo MACEDO ${ }^{3}$; Paulo Boller GALLO \\ Paulo de Souza GONÇALVES ${ }^{5}$; José Nivaldo GARCIA ${ }^{6}$; Eduardo Luiz LONGUI7; \\ Miguel Luiz Menezes FREITAS ${ }^{8}$; Alexandre Magno SEBBENN ${ }^{2}$
}

RESUMO - Hevea brasiliensis (Willd. ex Adr. Juss.), a seringueira, é utilizada para a produção de látex e, ao final do ciclo produtivo, a madeira também pode ser aproveitada. Dessa maneira, o melhoramento genético visa aumentar a produção tanto de látex quanto de madeira. Este estudo teve por objetivo determinar a variabilidade genética, o controle genético de caracteres de crescimento e a seleção de clones para a região de Mococa, Estado de São Paulo. Um teste clonal foi instalado no município de Mococa, com delineamento experimental de blocos ao acaso, com oito tratamentos (clones), três repetições e 12 plantas por parcela, utilizando-se o espaçamento entre plantas de 7 × $2 \mathrm{~m}$. Os caracteres avaliados foram: diâmetro à altura do peito - DAP, altura total - ALT, altura da bifurcação - AB e volume real individual de madeira - VOL. Diferenças significativas foram detectadas entre os clones para todos os caracteres, o que indica a possibilidade de melhoramento pela seleção. $\mathrm{O}$ coeficiente de herdabilidade no sentido amplo foi alto $(0,72)$ para $\mathrm{AB}$ e baixo para DAP e VOL. O coeficiente de correlação genética foi alto, positivo e significativo para os caracteres DAP x ALT, DAP x VOL e ALT x VOL, o que indica que a seleção em um caráter pode trazer ganhos indiretos em outro. Os resultados mostraram que a seleção de clones com maior DAP pode resultar no aumento da ALT e VOL de plantios comerciais da espécie. Pode-se destacar o clone RRIM701, proveniente da Malásia, como o mais indicado para todos os caracteres de crescimento, bem como para o cultivo em Mococa-SP.

Palavras-chave: teste clonal; seleção; seringueira; melhoramento genético.

\begin{abstract}
Hevea brasiliensis (Willd. ex Adr. Juss.), locally known as seringueira, is used for latex production and at the end of production cycle, wood may be used for furniture. Thus, the breeding aimed at increasing the production of latex and wood. Our goal was to determine the genetic variability, genetic growth control of traits and selection of clones from Mococa region, São Paulo state. A clonal plantation was established in Mococa municipality, and experimental design used was randomized blocks, at a spacing of $7 \times 2 \mathrm{~m}$, eight treatments (clones), three replicates and 12 plants per plot. The evaluated traits were: diameter at breast height (DBH, $1.3 \mathrm{~m}$ from the ground), total height $-\mathrm{TH}$, bifurcation height $-\mathrm{BH}$ and individual real wood volume - VOL. Significant differences were detected between clones for all traits, indicating the possibility of improvement by selection. Broad-sense heritability coefficient was high (0.72) for BH and low to DBH and VOL. Genetic correlation coefficient was high, positive and significant for the traits (TH $\times$ DBH, DBH $\times$ VOL and TH $x$ VOL, which indicates that selection on a trait may results gains in another trait. Results showed that clones selection with higher DBH may results in increase of TH and VOL in commercial plantation. We emphasize that RRIM701 clone, from Malaysia, is the most appropriate for all growth traits for growing in Mococa-SP.
\end{abstract}

Keywords: clonal test; selection; rubber tree; genetic improvement.

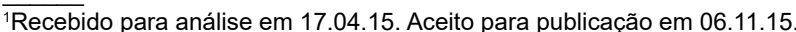

${ }^{2}$ Instituto Florestal, Rua do Horto 931, 02377-000 São Paulo, SP, Brasil.

${ }^{3}$ Mestrando em Agronomia, Sistemas de Produção, FEIS/UNESP. hugoengenheiro@yahoo.com.br

${ }^{4}$ Instituto Agronômico, Programa Seringueira, Caixa Postal 28, 13020-970 Campinas, SP, Brasil. paulogallo@apta.sp.gov.br

${ }^{5}$ Instituto Agronômico, Programa Seringueira, Caixa Postal 28, 13020-970 Campinas, SP, Brasil. paulog@iac.sp.gov.br

${ }^{6}$ Departamento de Ciências Florestais da ESALQ -USP, Universidade de São Paulo, Caixa Postal 9, 13400-970 Piracicaba, SP, Brasil. jngarcia@esalq.usp.br

${ }^{7}$ Instituto Florestal, Rua do Horto 931, 02377-000 São Paulo, SP, Brasil. elongui@if.sp.gov.br

IInstituto Florestal, Rua do Horto 931, 02377-000 São Paulo, SP, Brasil. miguellmfreitas@yahoo.com.br

${ }^{9}$ Autor para correspondência: Israel Luiz de Lima - israelluizde.lima@yahoo.com.br
} 


\section{INTRODUÇÃO}

Hevea brasiliensis (Willd. ex Adr. Juss.), planta da família Euphorbiaceae, conhecida como seringueira, é uma espécie arbórea de grande importância econômica para o Brasil, pela produção de látex e madeira (Furlani et al., 2005). De acordo com o International Rubber Study Group - IRSG, a área plantada de $H$. brasiliensis, no Brasil, em 2010, foi de 169,5 mil hectares (IRSG, 2012). Segundo Lorenzi (1992), a espécie tem sua ocorrência natural restrita à Amazônia brasileira (latitude $7^{\circ} \mathrm{N}$ a $15^{\circ} \mathrm{S}$ ), mas é cultivada atualmente em diversas regiões do País, como nos estados de São Paulo, Espírito Santo, Mato Grosso, Mato Grosso do Sul, Goiás, Bahia e Paraná (Gonçalves e Marques, 2008), além de outros países, tais como Vietnã, Malásia e Indonésia; portanto, o Brasil tem potencial para aumentar a produção de borracha. Somente o Estado de São Paulo possui 14 milhões de hectares que podem ser utilizados pela heveicultura (Instituto Agronômico de Campinas - IAC, 2004). O Planalto Ocidental do estado engloba mais de $90 \%$ da área plantada, onde se situa a região mais importante de cultivo e que representa mais da metade de toda a área explorada com $H$. brasiliensis no Brasil (Gonçalves et al., 2011).

Uma forma de maximizar a utilização de H. brasiliensis é por meio da obtenção de produtos múltiplos. Na Ásia, desde o final do século passado, têm-se buscado algumas alternativas para elevar a renda do produtor que cultiva a espécie, tais como a produção de sementes, óleo, mel e uso da madeira. A exploração de madeira tem sido a alternativa complementar mais importante, extraída quando o período produtivo das árvores se encerra (Gonçalves et al., 2011).

A produtividade do látex e madeira de H. brasiliensis pode ser aumentada, utilizando-se do melhoramento genético de genótipos (ou materiais) que tenham potencial de utilização econômica (Kageyama et al., 2002). Programas de melhoramento genético de $H$. brasiliensis são direcionados pela seleção de indivíduos com alta produção e qualidade de borracha (Silva et al., 2014). A seleção é baseada principalmente em caracteres como taxa de crescimento, resistência a doenças e danos por ventos (Silva et al., 2014). No melhoramento genético de espécies arbóreas, é fundamental conhecer o controle genético dos caracteres de produtividade ou sua herdabilidade e a correlação fenotípica e genética entre estes caracteres para determinar o potencial de melhoramento genético indireto entre caracteres, além da interação genótipos-ambientes, ou seja, todos os elementos que afetam a seleção de genótipos superiores em termos de produtividade (Gonçalves et al., 2005; 2006; 2009; Gouvêa et al., 2013; Verardi et al., 2012; Silva et al., 2013; 2014).

$\mathrm{Na}$ avaliação de populações de Hevea, o perímetro do caule das árvores é o parâmetro mais importante de decisão do grau de maturidade da plantação para a colheita do látex, podendo ser também um parâmetro indireto de seleção de material para serraria (Krishan, 2015).

Nesse contexto, os objetivos deste estudo foram determinar o controle genético de caracteres de crescimento, a associação fenotípica e genética entre os caracteres e a seleção de clones de H. brasiliensis para a região de Mococa, Estado de São Paulo, com o propósito de aumentar a produtividade de madeira.

\section{MATERIAL E MÉTODOS}

O presente estudo foi conduzido em um teste clonal instalado em 1989 no município de

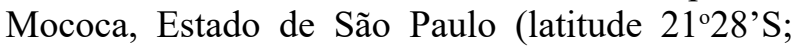
longitude $47^{\circ} 01$ ' $\mathrm{E}$; altitude de $665 \mathrm{~m}$ ). A temperatura média anual do local é de $24,5{ }^{\circ} \mathrm{C}$ e a precipitação anual média é de $1.500 \mathrm{~mm}$. O delineamento experimental utilizado foi o de blocos ao acaso, com os oito tratamentos (clones), três repetições (blocos) e 12 plantas úteis por parcela. O espaçamento utilizado foi o 7 x $3 \mathrm{~m}$. Os oito clones utilizados são originários de diferentes países: clone IAN873, Brasil; clones GT1, PR261 e PR255, Indonésia; clones RRIM701, RRIM600, PB217 e PB235, Malásia (Tabela 1). O clone IAN873 foi desenvolvido pelo antigo Instituto Agronômico do Norte (IAN), atual Embrapa Amazônia Oriental. Os clones GT1, PR261 e PR255 são provenientes das estações experimentais Gondang Tapen e Proefstation voor Rubber, na Indonésia, e os clones RRIM701, RRIM600, PB217 e PB235 da Malásia são originais do Rubber Research Institute of Malaysia e das plantações de Prang Besar, do grupo Harisson and Crossifield. O teste clonal foi mensurado aos 19 anos de idade para os caracteres diâmetro à altura do peito - DAP, altura total - ALT e altura da bifurcação - AB. $\mathrm{O}$ volume real - VOL foi calculado pela expressão: $V O L=\left[\pi(D A P)^{2} A L T x F F\right] / 4$, em que FF é o fator de forma para a espécie, de acordo com Oliveira et al. (2000). 
LIMA, I.L. de et al. Seleção de clones de Hevea brasiliensis

Tabela 1. Origem parental e país de origem dos clones de Hevea brasiliensis.

Table 1. Parental and country origin of Hevea brasiliensis clones.

\begin{tabular}{lcc}
\hline Clone & Parentesco de origem & País de origem \\
\hline IAN873 & PB86 x FA1717 & Brasil \\
GT1 & Clone primário & Indonésia \\
PR261 & Tjir1 x PR107 & Indonésia \\
PR255 & Tjir1 x PR107 & Indonésia \\
PB217 & PB5/51 x PB69 & Malásia \\
RRIM701 & $44 / 553 \times$ RRIM501 & Malásia \\
PB235 & PB5/51 x PBS78 & Malásia \\
RRIM 600 & Tjir1 x PB69 & Malásia \\
\hline
\end{tabular}

As análises de variância para os caracteres foram realizadas com o auxílio do programa SAS (SAS, 1999), seguindo o modelo estatístico:

$$
Y_{i j}=\mu+b_{i}+T_{j}+e_{i j},
$$

em que: $Y_{i j}$ é o valor fenotípico do $j$-ésimo indivíduo da $i$-ésima repetição; $\mu$ é o termo fixo da média total; $b_{i}$ é o efeito fixo da $i$-ésima repetição (bloco); $T_{j}$ é o efeito aleatório do $j$-ésimo clone; $e_{i j}$ é o efeito aleatório da interação entre o $j$-ésimo clone e a $i$-ésima repetição (erro entre parcelas). Sendo, $i=1 \ldots .$. b (b é o número de repetições); $j=1 . . . \mathrm{t}$ ( $\mathrm{t}$ é o número de clone). Para verificar se as médias dos caracteres eram significativamente diferentes entre os clones, utilizou-se o teste de Tukey, assumindo, neste caso, como sendo fixo o efeito de clones.

Da análise de variância dos caracteres individuais foram estimados os componentes de variância genética entre clones $\left(\sigma_{c}^{2}\right)$, variância ambiental $\left(\sigma_{e}^{2}\right)$ e variância fenotípica entre clones $\left(\sigma_{F}^{2}=\sigma_{c}^{2}+\sigma_{e}^{2}\right)$. Os coeficientes de variação experimental $\left(C V_{e}\right)$ e genética entre clones $\left(C V_{g}\right)$ foram estimados pelas expressões:

$$
\begin{aligned}
& C V_{e}=100\left(\frac{\sigma_{e}^{2}}{x}\right) \mathrm{e} \\
& C V_{g}=100\left(\frac{\sigma_{c}^{2}}{x}\right),
\end{aligned}
$$

em que $x$ é a média do caráter analisado.
O coeficiente de herdabilidade no sentido amplo $(H)$ foi estimado pela expressão:

$$
H=\left(\frac{\sigma_{c}^{2}}{\sigma_{F}^{2}}\right) .
$$

As correlações fenotípicas $\left(r_{F}\right)$ e genéticas $\left(r_{g}\right)$ entre os caracteres foram estimadas a partir dos valores individuais, de acordo com as seguintes expressões:

$$
\begin{gathered}
r_{F}=\frac{\sigma_{F_{X} F_{Y}}}{\sqrt{\sigma_{F_{X}}^{2} \cdot \sigma_{F_{Y}}^{2}}} \mathrm{e}, \\
r_{g}=\frac{\sigma_{p_{X} p_{Y}}}{\sqrt{\sigma_{p_{X}}^{2} \cdot \sigma_{p_{Y}}^{2}}},
\end{gathered}
$$

em que: $r_{F}$ e $r_{g}$ são os coeficientes de variação fenotípica e genética; $\sigma_{F_{X} F_{Y}}$ e $\sigma_{p_{X} p_{Y}}$ são os produtos cruzados fenotípicos e genéticos dos caracteres x e y, estimados a partir das análises de covariância; $\sigma_{F_{X}}^{2}$ e $\sigma_{p_{X}}^{2}$ são as variâncias fenotípicas dos caracteres x e y, respectivamente, e $\sigma_{F_{Y}}^{2}$ e $\sigma_{P_{Y}}^{2}$ são as variâncias genéticas dos caracteres $\mathrm{x}$ e y, respectivamente (Falconer, 1987).

\section{RESULTADOS E DISCUSSÃO}

A análise de variância revelou diferenças significativas entre clones pelo teste $\mathrm{F}$ a $1 \%$ de probabilidade para todos os caracteres estudados, o que sugere a possibilidade de seleção de clones e o melhoramento genético (Tabela 2). 
Tabela 2. Resultados do teste $\mathrm{F}$ da análise de variância para os quadrados médios, média, coeficiente de variação experimental $\left(C V_{e}\right)$ e coeficiente de variação genética $\left(C V_{g}\right)$ para diâmetro à altura do peito - DAP, altura total - ALT, altura da bifurcação - AB e volume real - VOL em teste clonal de Hevea brasiliensis.

Table 2. Results of $\mathrm{F}$ test of the analysis of variance for mean squares, mean, coefficient of experimental variation $\left(C V_{e}\right)$ and coefficient of genetic variation $\left(C V_{g}\right)$ for diameter at breast height - DAP, total height - ALT, fork height - AB and real volume -VOL in a Hevea brasiliensis clonal test.

\begin{tabular}{cccccc}
\hline & & \multicolumn{4}{c}{ Quadrado médio } \\
\cline { 3 - 5 } Fonte de variação & GL & DAP & ALT & AB & VOL \\
& & $(\mathrm{cm})$ & $(\mathrm{m})$ & $(\mathrm{m})$ & $\left(\mathrm{m}^{3} /\right.$ árv. $)$ \\
\hline Blocos & 2 & 6,01 & 90,56 & 20,28 & 0,15 \\
Clone & 7 & $42,91^{* *}$ & $8,92^{* *}$ & $74,55^{* *}$ & $0,43^{* *}$ \\
\hline Média & 23,32 & 22,56 & 6,57 & 0,91 \\
$C V_{e}(\%)$ & 15,6 & 22,6 & 47,9 & 39,0 \\
$C V_{g}(\%)$ & 6,1 & 4,1 & 2,9 & 18,1 \\
\hline
\end{tabular}

Em que: $\mathrm{GL}=$ graus de liberdade; $* * \mathrm{P}>0,01$.

Where: $\mathrm{GL}=$ degrees of freedom; $* * \mathrm{P}>0.01$.

Contudo, o teste de Tukey indicou que essas diferenças significativas se devem a diferenças entre as médias de crescimento entre os clones PB217 e RRIM701 em relação ao PB235 para DAP, PR261, PR255 e
RRIM701 em relação ao RRIM600 para ALT, PR261 e RRIM701 em relação aos clones IAN873 e PB217 para AB e RRIM701 em relação aos clones PB217, PB235 e RRIM600 para VOL (Tabela 3).

Tabela 3. Médias para diâmetro à altura do peito - DAP), altura total - ALT, altura da bifurcação - AB e volume real - VOL em clones de Hevea brasiliensis, aos 19 anos de idade.

Table 3. Average of diameter at breast height - DAP, total height - ALT, fork height - AB and real volume -VOL of Hevea brasiliensis clone at 19-year-old.

\begin{tabular}{lcccc}
\hline Clone & DAP $(\mathrm{cm})$ & ALT $(\mathrm{m})$ & AB $(\mathrm{m})$ & VOL $\left(\mathrm{m}^{3} /\right.$ árv. \\
\hline PB217 & $24,55^{\mathrm{a}}$ & $20,02^{\mathrm{ab}}$ & $5,03^{\mathrm{b}}$ & $0,97^{\mathrm{b}}$ \\
GT1 & $23,81^{\mathrm{ab}}$ & $19,33^{\mathrm{ab}}$ & $7,24^{\mathrm{ab}}$ & $0,89^{\mathrm{ab}}$ \\
PR261 & $22,95^{\mathrm{ab}}$ & $8,54^{\mathrm{a}}$ & $0,95^{\mathrm{a}}$ & $0,93^{\mathrm{ab}}$ \\
PR255 & $21,06^{\mathrm{a}}$ & $6,53^{\mathrm{ab}}$ & $0,84^{\mathrm{ab}}$ \\
IAN873 & $24,07^{\mathrm{ab}}$ & $20,31^{\mathrm{ab}}$ & $5,07^{\mathrm{b}}$ & $1,10^{\mathrm{a}}$ \\
RRIM701 & $22,53^{\mathrm{ab}}$ & $22,10^{\mathrm{a}}$ & $8,86^{\mathrm{a}}$ & $0,75^{\mathrm{b}}$ \\
PB235 & $24,80^{\mathrm{a}}$ & $18,66^{\mathrm{ab}}$ & $6,59^{\mathrm{ab}}$ & $0,76^{\mathrm{b}}$ \\
RRIM600 & $21,66^{\mathrm{b}}$ & $18,39^{\mathrm{b}}$ & $5,08^{\mathrm{ab}}$ & \\
\hline
\end{tabular}

Observação: médias seguidas da mesma letra não diferem significativamente entre si a $5 \%$ de probabilidade pelo teste de Tukey.

Note: Means followed by the same letter do not differ significantly from each other at $5 \%$ probability by Tukey test. 
O clone RRIM701 apresentou a maior média para todos os caracteres e os clones PB235 e RRIM600 apresentaram os menores crescimentos em DAP e VOL; logo, o clone que apresentou melhor desempenho para todos os caracteres foi o RRIM701, proveniente da Malásia, sendo o mais indicado para reflorestamentos comerciais para a produção de madeira no local de estudo.

De acordo com Gonçalves et. al. (1991), no Estado de São Paulo, os clones RRIM600, RRIM701, GT1, PB235, PR107 e IAN873 são os mais utilizados em plantios comerciais. Entre esses, os clones RRIM600 e GT1 apresentam maior desenvolvimento silvicultural e maior disponibilidade no mercado atual. Entretanto, estima-se que o clone RRIM600 seja o mais plantado, com cerca de $80 \%$ da área paulista utilizada para o cultivo de seringueira (Gonçalves, 2010). De Jesus et al. (2015), em um estudo com os principais clones comerciais utilizados, atualmente, no Brasil (RRIM600 e GT1), constaram que os mesmos têm aplicações em pequenas estruturas, construção leve, e indústria de móveis e podem ser uma alternativa no mercado brasileiro para reduzir a demanda por madeira de espécies florestais nativas.

Segundo Simmonds (1989), em plantas mais baixas em altura e com internódios mais curtos ocorre maior produção de látex e ainda há menores danos ocasionados pelo vento, bem como maior facilidade na colheita.

O coeficiente de variação experimental foi de média magnitude para o DAP $(15,6 \%)$ e $\operatorname{ALT}(11,6 \%)$ e alto para AB $(47,9 \%)$ e VOL $(39,0 \%)$, indicando baixa precisão experimental para os dois últimos caracteres (Tabela 2). O coeficiente de variação genético foi alto para o caráter $\operatorname{VOL}(18,1 \%)$, mas baixo para os demais caracteres (máximo de $6,1 \%$ para o DAP). Embora esse coeficiente tenha sido baixo para DAP, ALT e AB, indica que existe variação genética entre os clones, e estes clones podem ser utilizados em programas de melhoramento e, ainda assim, apresentar ganhos indiretos na produção de madeira.

O coeficiente de herdabilidade mede o controle genético ou o quanto da variação fenotípica de caracteres quantitativos ou qualitativos é de origem genética. A estimativa do coeficiente de herdabilidade no sentido amplo (Tabela 4) foi alta para $\mathrm{AB}(=0,719)$, média para $\mathrm{DAP}(=0,300) \mathrm{e}$ $\operatorname{VOL}(=0,240)$ e baixa para ALT $(=0,004)$; logo, o caráter $\mathrm{AB}$ apresenta forte controle genético e sugere a possibilidade de se obter progressos genéticos com a seleção entre esses oito clones, seguida do DAP e do VOL.

Tabela 4. Estimativa do coeficiente de herdabilidade no sentido amplo $(H)$ e variância ambiental $\left(\sigma_{e}^{2}\right)$ para os caracteres diâmetro à altura do peito - DAP, altura total - ALT, altura da bifurcação - AB e volume real - VOL em clones de Hevea brasiliensis.

Table 4. Estimate of the coefficient of broad sense heritability $(H)$, environmental variance $\left(\sigma_{e}^{2}\right)$ to diameter at breast height - DAP, total height - ALT, fork height - AB and real volume - VOL in Hevea brasiliensis clones.

\begin{tabular}{ccccc}
\hline Parâmetro & DAP & ALT & AB & VOL \\
\hline$H$ & 0,300 & 0,004 & 0,719 & 0,205 \\
$\sigma_{e}^{2}$ & 0,005 & 0,202 & 0,005 & 0,068 \\
\hline
\end{tabular}

As estimativas do coeficiente de correlação genética foram todas positivas entre os pares de caracteres (mínimo de 0,35), embora significativas apenas para DAP x ALT, DAP x VOL e ALT x VOL (Tabela 5).

As estimativas da correlação fenotípica entre pares de caracteres foram também positivas, porém menores que as correlações genéticas e não significativas. Associações genéticas positivas entre caracteres indicam que a seleção em um carácter pode trazer ganhos indiretos em outro (Falconer, 1987). Como as maiores correlações genéticas encontradas foram entre DAP x VOL $(0,85)$ e ALT x VOL $(0,86)$, e como o DAP é um carácter medido com maior precisão, a seleção com base no DAP resultará em aumento do VOL, bem como a ALT da população selecionada. 
LIMA, I.L. de et al. Seleção de clones de Hevea brasiliensis

Tabela 5. Estimativas das correlações genéticas $\left(r_{g}\right)$ e fenotípicas $\left(r_{F}\right)$ em clones de Hevea brasiliensis.

Table 5. Estimates of genetic $\left(r_{g}\right)$ and phenotypic correlations $\left(r_{F}\right)$ in Hevea brasiliensis clones.

\begin{tabular}{lcc}
\hline Caracteres & $r_{g}$ & $r_{F}$ \\
\hline DAP x ALT & $0,81^{*}$ & 0,53 \\
DAP x VOL & $0,85^{*}$ & 0,43 \\
DAP x AB & 0,42 & 0,03 \\
ALT x VOL & $0,86^{*}$ & 0,37 \\
ALT x AB & 0,67 & 0,28 \\
VOL x AB & 0,35 & 0,26 \\
$*$ P $<0,05$, DAP = diâmetro à altura do peito; ALT = altura total; $\mathrm{AB}=$ altura da bifurcação; $\mathrm{VOL}=$ volume real. \\
$* \mathrm{P}<0.05, \mathrm{DAP}=$ diameter at breast height; ALT = total height; $\mathrm{AB}=$ fork height; $\mathrm{VOL}=$ real volume.
\end{tabular}

\section{CONCLUSÕES}

Existe variação genética para os caracteres entre os clones estudados.

O carácter altura de bifurcação apresenta alto controle genético; portanto, apresenta maior potencial para a seleção.

Os caracteres DAP, ALT, AB e VOL são positivamente correlacionados; logo, a seleção para qualquer um destes resulta em aumento indireto nos outros.

O clone RRIM701, proveniente da Malásia, é o mais indicado para todos os caracteres de crescimento simultaneamente, para o cultivo em Mococa-SP.

\section{AGRADECIMENTOS}

Os autores agradecem a Sonia R. G. Campião e Francisco Bianco pelo auxílio na mensuração do experimento. Os autores Alexandre Magno Sebbenn, Miguel Luiz Menezes Freitas, Eduardo Luiz Longui e Israel Luiz de Lima agradecem ao Conselho Nacional de Desenvolvimento Científico e Tecnológico - CNPq pela concessão da bolsa de Produtividade em Pesquisa.

\section{REFERÊNCIAS BIBLIOGRÁFICAS}

DE JESUS, E.J.H. et al. Potential of rubberwood (Hevea brasiliensis) for structural use after the period of latex extraction: a case study in Brazil. Journal of Wood Science (Print), v. 61, p. 384-390, 2015.
FALCONER, D.S. Introdução à genética quantitativa. Viçosa, MG: UFV, 1987. 279 p.

FURLANI, R.C.M. et al. Mating system in a Hevea brasiliensis population by isozyme loci. Crop Breeding and Applied Biotechnology, v. 5, p. 402-409, 2005.

GONÇALVES, P. de S. Clones de seringueira: recomendação para plantio no estado de São Paulo. In: CICLO DE PALESTRAS SOBRE HEVEICULTURA PAULISTA, 7., 2010, São José do Rio Preto. Palestras... São José do Rio Preto: APABOR, 2010. 8 p.

. et al. Performance of new Hevea clones from IAC 400 series. Scientia Agricola, v. 64, p. 241-248, 2011.

. et al. Clones de Hevea: influência dos fatores ambientais na produção e recomendação para o plantio. Campinas: IAC, 1991. 32 p. (Boletim Técnico, 138).

et al. Genetic variability and

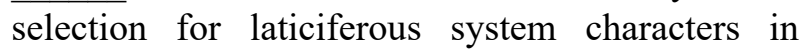
Hevea brasiliensis. Genetic and Molecular Biology, v. 28, p. 414-422, 2005.

et al. Genetic variability for girth $\overline{\text { growth }}$ and rubber yield characters in Hevea brasiliensis. Science Agricultural, v. 63, p. 246-254, 2006.

. et al. Genetic variation and realized genetic gain from rubber tree improvement. Science Agriculture, v. 66, p. 44-51, 2009. 
GONÇALVES, P. de S.; MARQUES, J.R.B. Melhoramento genético da seringueira: passado, presente e futuro. In: ALVARENGA, A.P.; CARMO, C.A.F.S. (Ed.). Seringueira. Viçosa, MG: Epamig, 2008. p. 399-534.

GOUVÊA, L.R.L. et al. Rubber tree early selection for yield stability in time and among locations. Euphytica, v. 191, p. 365-373, 2013.

INSTITUTO AGRONÔMICO DE CAMPINAS - IAC. Programa Seringueira. 31/8/2004. Disponível em: $<$ http://www.iac.sp.gov.br/centros/centro_cafe/ seringueira/programa.htm>. Acesso em: 10 ago. 2014.

INTERNATIONAL RUBBER STUDY GROUP IRSG. Area under plantation rubber (Table 28). Rubber Statistical Bulletin, v. 66, n. 7-8, 2012.

KAGEYAMA, P. Y. et al. Ganhos na seleção para a produtividade de látex em população natural de Hevea brasiliensis na Reserva Chico Mendes: estudo de caso das IAPs (Ilhas de alta produtividade). Scientia Forestalis, n. 61, p. 79-85, 2002.

KRISHAN, B. Growth assessment of popular clones of natural rubber (Hevea brasiliensis) under warm dry climatic conditions of Chattisgarh State, Central India. Journal of Experimental Biology and Agricultural Sciences, v. 3, n. 2, p. 157-161, 2015.

LORENZI, H. Árvores brasileiras: manual de identificação e cultivo de plantas arbóreas nativas do Brasil. Nova Odessa: Plantaraum, 1992. $302 \mathrm{p}$.

OLIVEIRA, S.A. et al. Variação genética em progênies de aroeira (Myracrodruon urundeuva Fr. All.) sob diferentes condições de cultivo. I - Aspectos silviculturais. Revista Instituto Florestal, v. 12, n. 2, p. 155-166, 2000.

SAS INSTITUTE INC. SAS Procedures Guide. Version 8 (TSMO). Cary: SAS Institute Inc., 1999.

SILVA, G.A.P. et al. Genetic parameters and correlation in early measurement cycles in rubber trees. Euphytica, v. 189, p. 343-350, 2013.

. et al. Genetic parameters in a rubber tree population: heritabilities, genotype-by-environment interactions and multi-trait correlations. Tree Genetics \& Genomes, v, 10, n. 6, p. 1511-1518, 2014. DOI:10.1007/S11295-014-0766-5.
SIMMONDS, N.W. Rubber breeding. In: WEBSTER, C.C.; BAULKWILL, W.J. (Ed.). Rubber. London: Longman, 1989. p. 85-124.

VERARDI, C.K. et al. Estimation of genetic parameters in rubber progenies. Crop Breed Applied Biotechnology, v. 12, p. 185-190, 2012. 